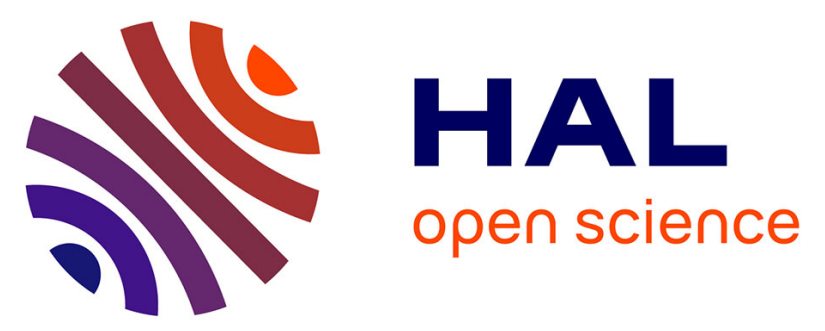

\title{
Plasmonics: influence of the intermediate (or stick) layer on the efficiency of sensors
}

Dominique Barchiesi, Demetrio Macías, L. Belmar-Letellier, D. van Labeke, Marc Lamy de La Chapelle, Timothée Toury, Eric Kremer, Laurence Moreau, Thomas Grosges

\section{To cite this version:}

Dominique Barchiesi, Demetrio Macías, L. Belmar-Letellier, D. van Labeke, Marc Lamy de La Chapelle, et al.. Plasmonics: influence of the intermediate (or stick) layer on the efficiency of sensors. Applied Physics B - Laser and Optics, 2008, 93 (1), pp.177-181. 10.1007/s00340-008-3173-5 . hal-00369408

\section{HAL Id: hal-00369408 \\ https://hal.science/hal-00369408}

Submitted on 11 May 2021

HAL is a multi-disciplinary open access archive for the deposit and dissemination of scientific research documents, whether they are published or not. The documents may come from teaching and research institutions in France or abroad, or from public or private research centers.
L'archive ouverte pluridisciplinaire HAL, est destinée au dépôt et à la diffusion de documents scientifiques de niveau recherche, publiés ou non, émanant des établissements d'enseignement et de recherche français ou étrangers, des laboratoires publics ou privés. 


\title{
Plasmonics: influence of the intermediate (or stick) layer on the efficiency of sensors
}

\author{
D. Barchiesi, D. Macías, L. Belmar-Letellier, D. van Labeke, M. Lamy de la Chapelle, T. Toury, E. Kremer, \\ L. Moreau, T. Grosges
}

\begin{abstract}
The efficiency of plasmon-resonance-based sensors can be greatly improved with an accurate design of the nano-structures that constitute them. In this work we focus our attention on the design of a particular kind of these nanometric structures that consists of planar multilayered systems. We show the significant influence, on the plasmon resonance conditions, of the intermediate layer used to adhere a thin film of gold to a dielectric substrate. To illustrate this effect, we consider different intermediate layers to compute the optimal geometry that vanish the reflected energy. For this, we use the S-formulation and an evolutionary method for the electromagnetic and the optimization problems, respectively.
\end{abstract}

D. Barchiesi $(\varangle) \cdot$ L. Moreau · T. Grosges

Project Group for Automatic Mesh Generation and Adaptation Methods, Gamma Project, University of technology of Troyes, 12 rue Marie Curie, 2060, 10010 Troyes cedex, France e-mail: dominique.barchiesi@utt.fr

D. Macías · T. Toury $\cdot$ E. Kremer

Project Group for Nanotechnology and Optical Instrumentation, Charles Delaunay's Institute FRE CNRS 2848, University of technology of Troyes, 12 rue Marie Curie, 2060, 10010 Troyes cedex, France

L. Belmar-Letellier · D. van Labeke

Optical Department of FEMTO UMR CNRS 6174, University of

Franche-Comté, 13 route de Gray, 2500 Besançon cedex, France

Present address:

L. Belmar-Letellier

ITAAPY, 9 rue Darwin, 75018 Paris, France

M. Lamy de la Chapelle

Laboratoire de Biophysique Moléculaire, Cellulaire et Tissulaire (BioMoCeTi) UMR CNRS 7033, University of Paris XIII, UFR Santé, Médecine et Biologie Humaine, 74 rue Marcel Cachin, 93017 Bobigny cedex, France
PACS $78.40 . \mathrm{Kc} \cdot 41.20 . \mathrm{-q} \cdot 02.60 . \mathrm{Pn} \cdot 61.46 .+\mathrm{w}$

\section{Introduction}

Since the early developments of the Near-Field optical microscopies, their wide variety of applications has not ceased to grow. Furthermore, a fairly recent area of the nanotechnologies with applications ranging from telecommunications to biologic-sensors has appeared: The plasmonics. All the associated components of surface-plasmon resonance sensors are made of metal structured settlements that are deposited on dielectric, and many are illuminated in reflection, through the dielectric sample, with p-polarized wave, in the Kretschmann configuration [1]. The active metallic region is made of gold but is deposited on an another metal thin layer to ensure adherence. Historically, the influence of this intermediate layer has been rarely considered and direct optimization has been made just with a gold layer [2]. Nevertheless, the dependence of the optical properties of such metallic structures on size and material choice still is an active area of researches. Moreover, the quality of the SPR sensor depends strongly on these parameters and nanosensors have a more complex behavior as the number of degrees of freedom increases [3-5].

It has been experimentally observed that the $\mathrm{Cr}$ interlayer influences the structural and optical properties of $\mathrm{Ag}$ films. The grain sizes and the reflectivity of $\mathrm{Ag}$ film in the $\mathrm{Ag} / \mathrm{Cr} / \mathrm{glass}$ system increase [6]. The influence of the intermediate Chromium layer has been studied in Refs. [7, 8]. The presence of a thin chromium adhesion layer shifts and broadens the surface plasmon resonance (SPR) shape. The magnitude of the resonance decreases when a thin film of chromium or a thin film of gold is employed. Its linear range 
becomes narrower as the thickness of the metal films increases. In Ref. [9], authors demonstrate that titanium is preferred over chromium as an adhesion layer since titanium is less strongly absorbing.

In the present work, we not only show that this thin intermediate layer may influence the quality of the plasmon resonance, but also that both intermediate and gold layer thicknesses must be computed simultaneously to get a more efficient coupling between the light and the metallic layers. The best gold layer thickness is shown to differ from the commonly used value of $50 \mathrm{~nm}$. We use an evolutionary procedure to search for the optimal plasmonic efficiency of the system studied. This is done in the visible range, through the design of the whole system (gold and intermediate layer thicknesses, as well as the angle of incidence of the illuminating light) in the visible range, for the best plasmon efficiency.

The paper is organized as follows. Section 2 is devoted to the description of the multilayer setup. In Sect. 3, we introduce the used computational model. In Sect. 4, numerical results are presented and discussed, before concluding in Sect. 5 .

\section{The multilayered setup}

The purpose of this study is to design a nano-sensor with maximal SPR efficiency, through the minimization of the reflected light. For this, we consider the nano-sensor as the multilayered system shown in Fig. 1. The material parameters are the relative permittivities $\epsilon_{1}=2.25$ (glass), $\epsilon_{2}\left(\lambda_{0}\right)$ (for chromium, tungsten or silver), $\epsilon_{3}\left(\lambda_{0}\right)$ (gold) and $\epsilon_{4}=$ 1.77 [10]. The illuminating field is a p-polarized plane wave of wavelength $\lambda_{0}$ (in vacuum) and angle of incidence $\theta_{i}$.

Our goal is to find the optimal set of parameters $\mathbf{p}=$ $\left(e_{1}, e_{2}, \theta_{i}\right)$ that minimizes the reflection coefficient $R / I_{0}$, where $I_{0}$ is the incident intensity. If this minimum is close to zero, it corresponds to a maximal transfer of energy of the wave illuminating the metallic setup and therefore to a plasmon excitation. The plasmon has been described as the trace of the pole of the reflected intensity along the real axis in the complex plane [11]. Also, this point has been fully discussed for metallic gratings in Ref. [12]. Close to this pole, the computation of the electromagnetic field may present problems of convergence, and the blind use of classical methods of optimization can lead to convergence into another minimum not corresponding to the plasmon. Therefore, the Sformulation of the electromagnetic computation is required as well as a nonclassical optimization technique such as, for example, simulated annealing or evolutionary methods.

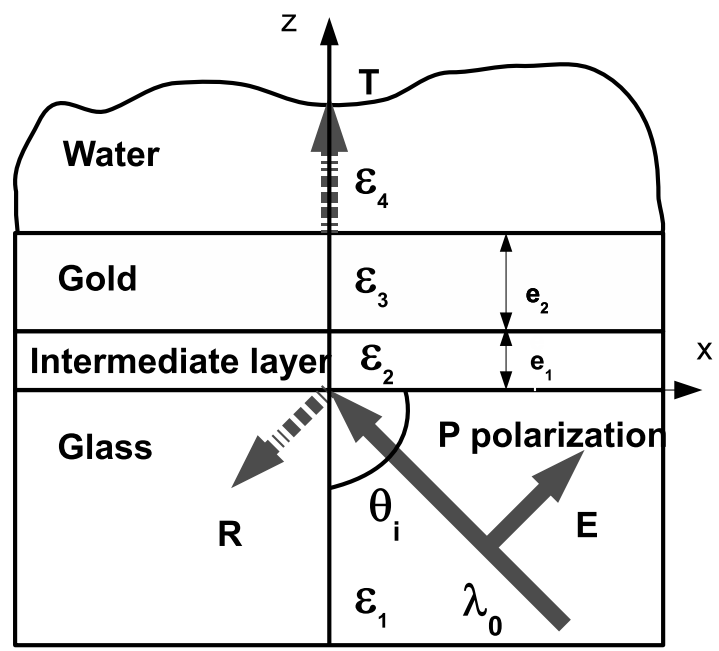

Fig. 1 Multilayered sample and illumination. The thicknesses of the intermediate and the gold layers are respectively $e_{1}$ and $e_{2}$. The detection is made in reflection and thus $R$ should be as small as possible. The incoming wave is p-polarized to generate surface plasmon and its wavelength is $\lambda_{0}$ in vacuum. The angle of incidence is $\theta_{i}$

\section{The computational model}

The computation of electromagnetic field interacting with planar interfaces has been studied for several decades [13, 14]. The reflected intensity is computed from the field reflected by the multilayered system shown in Fig. 1. The calculation of the resulting Fresnel coefficients is analytic, and the general method for $N$ materials, using the T-formulation can be found in Ref. [15]. Nevertheless, this formulation has proven to be numerically instable when the absorption and thickness increase [16]. In plasmonics, the accuracy of the numerical scheme must be carefully controlled due to the numerical instabilities related to the pole, especially if optimization is required. In Ref. [17], Vigoureux writes the reflection coefficient of a multilayered structure in terms of a polynomial development. Also, other efficient formulations have been extended from the theory of the diffraction of light by gratings [16, 18-20].

In the three plane interfaces problem under consideration, a simple analytical result may be used (Eq. 1), but the direct computation of the corresponding transmission coefficient $T / I_{0}$, where $I_{0}$ is the incident intensity, diverges when the thickness of the metal or the absorbance is increased. The direct computation of the reflection coefficient $R / I_{0}=\left|r_{14}\right|^{2}$ can then become instable near the resonance.

$r_{14}=\frac{r_{12}+r_{23} \exp \left(2 j e_{1} w_{2}\right)+r_{34} \exp \left(2 j\left(e_{2} w_{3}+e_{1} w_{2}\right)\right)+r_{12} r_{23} r_{34} \exp \left(2 j e_{2} w_{3}\right)}{1+r_{12} r_{23} \exp \left(2 j e_{1} w_{2}\right)+r_{12} r_{34} \exp \left(2 j\left(e_{1} w_{2}+e_{2} w_{3}\right)\right)+r_{23} r_{34} \exp \left(2 j e_{2} w_{3}\right)}$, 
where $r_{i j}$ are the Fresnel coefficients of the interface $i j$ :

$r_{i j}=\frac{\left(\epsilon_{j} w_{i}-\epsilon_{i} w_{j}\right)}{\left(\epsilon_{j} w_{i}+\epsilon_{i} w_{j}\right)}$

with $w_{i}=2 \pi \sqrt{\epsilon_{i}-u^{2}} / \lambda_{0}$ and $u=\sqrt{\epsilon_{1}} \sin \left(\theta_{i}\right)$. Nevertheless, the analytical formulation establishes a relation between the thicknesses $e_{1}$ and $e_{2}$, and the incidence angle $\theta_{i}$ if the zeros of $R$ are searched. For example, the gold thickness $e_{2}$ can be deduced from the intermediate layer thickness $e_{1}$ (see Fig. 1) through

$$
\begin{aligned}
e_{2}= & -j \log \left\{-\left[\exp \left(2 j w_{2} e_{1}\right) r_{23}+r_{12}\right]\right. \\
& \left./\left[\left(\exp \left(2 j w_{2} e_{1}\right)+r_{23} r_{12}\right) r_{34}\right]\right\} /\left(2 w_{3}\right) .
\end{aligned}
$$

This equation gives an infinity of complex thicknesses $e_{2}$ that have no physical signification. Moreover, the solution depends on both $\lambda_{0}$ and $\theta_{i}$. The expected solution for the thickness $e_{2}$ must be real, and therefore this formula will be used to test the optimization method.

Due to instabilities of the direct T-formulation of the electromagnetic problem, we use the S-formulation for plane multilayers. This allows the correct factorization of exponential functions to limit their influence on roundoff errors and to verify the energy conservation. The S-method and the algorithm have been fully described by $\mathrm{Li}$ [16]. The principle of the method lies on the factorization of the up and down exponentials that describe, in particular, the decrement of the skin depth of waves in metals [14]. The advantage of this formulation is its possibility to describe any $N$ absorbing multilayered geometry. An iterative scheme gives both reflected and transmitted fields (Fig. 1).

Among the nonclassical or last resort optimization methods, we have found that the Evolution Strategies provide an efficient solution to problems where classical methods fail [21] and particularly for the resolution of the inverse problem in nanotechnologies [22]. Their operational principles are based on the evolution of the searched parameters (objective variables), through the imitation of process of variation and selection that take place in natural evolution.

In the present study, the target is the minimal value of $R$. The basic steps of the evolutionary method can be summarized as follows: initialization of a random initial population of parameters $\mathbf{p}$ ( $\mu_{1}$ elements), random recombination ( $\mu_{2}>\mu_{1}$ elements), random mutation, selection of the $\mu_{1}$ best elements, and loop to the second step to reach the convergence of the $\mu_{1}$ elements of the $\mathbf{p}$ population [21]. Each iteration of the evolutionary loop corresponds to a generation of parameters.

\section{Results and discussion}

For our numerical experiments, we use $\mu_{2}=100$ and $\mu_{1}=14$ elements for the secondary and initial populations,

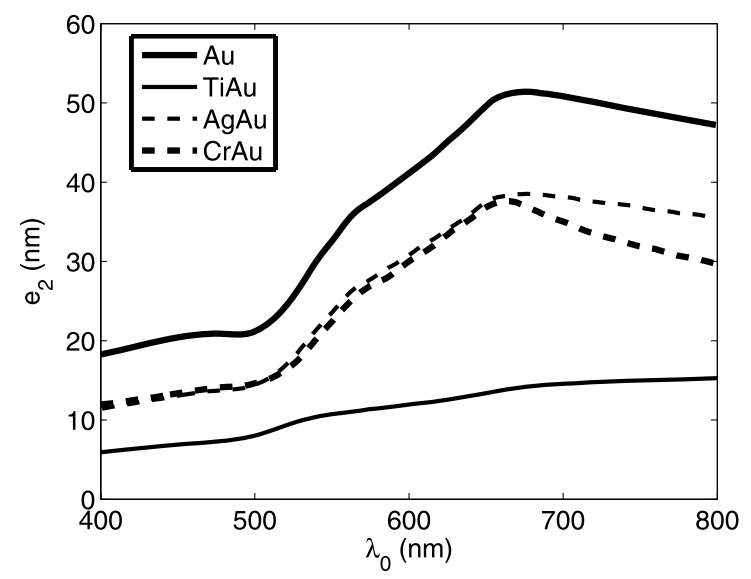

a

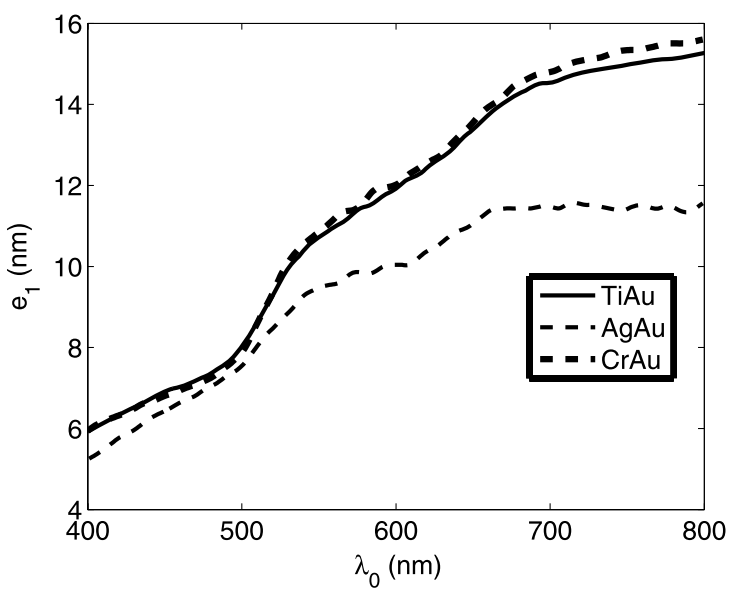

b

$e_{1}$

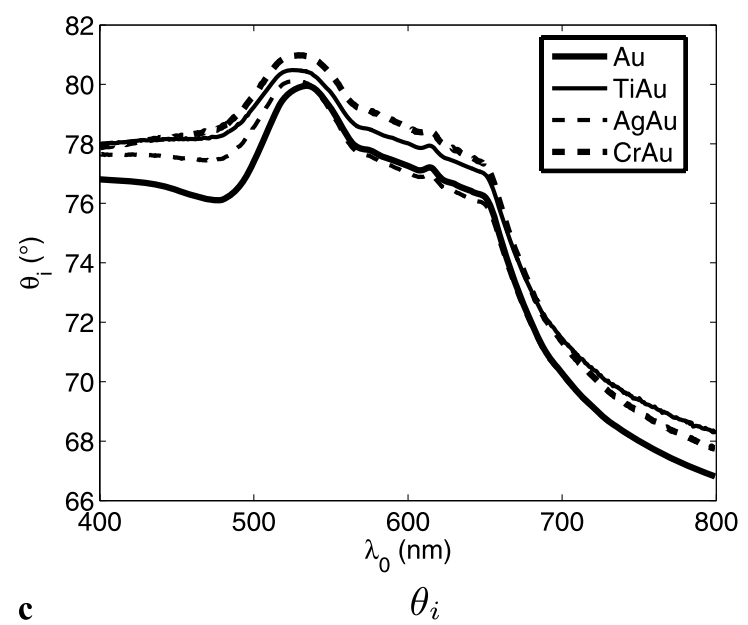

Fig. 2 The best values of the unknown parameters $\left(e_{2}, e_{1}, \theta_{i}\right)$ to optimize the plasmon resonance of multilayer metallic system deposited on glass, with water in the upper medium, as a function of the incoming wavelength

respectively. The required number of generations to reach convergence is less than 40 . At least 15 realizations of the whole evolutionary process are made to verify stability. That 

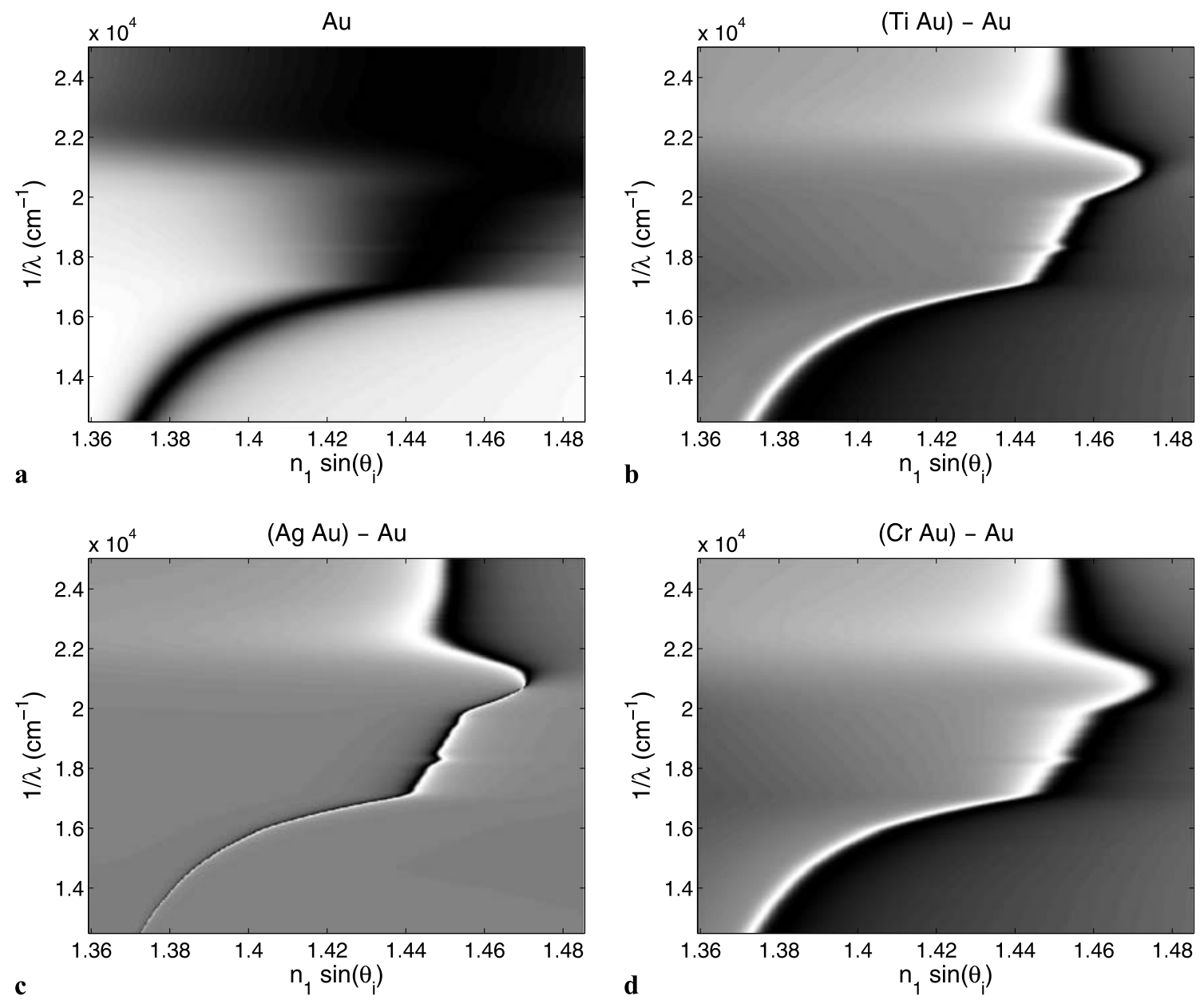

Fig. 3 Relative difference between the dispersion curves of gold (a) and gold with intermediate layer for $\mathrm{Ti}-\mathrm{Au}(\mathbf{b}), \mathrm{Ag}-\mathrm{Au}(\mathbf{c})$ and $\mathrm{Cr}-\mathrm{Au}(\mathbf{d})$, respectively: $C\left(u, \lambda_{0}\right)$. These differences are computed by using the best parameters $\left(e_{2}, e_{1}\right)$ for each wavelength (Fig. 2)

is, the search for the objective parameters starts from 15 different initial populations. The computed value of $R / I_{0}$ is less than $2 \times 10^{-11}$, corresponding to the total coupling of the incoming light with the metallic multilayered system. The transmission is also equal to 0 . Figure 2 shows the result of the optimization for $e_{2}$ (a), $e_{1}$ (b), and $\theta_{i}$ (c), as function of the wavelength in the whole visible domain, respectively. We compare the gold layer with and without $\mathrm{Cr}$, Ag, Ti intermediate layers.

Figure 2(a) shows that the optimal thickness of gold deposited on $\mathrm{Cr}$, $\mathrm{Ti}$, or $\mathrm{Ag}$ is smaller than this of gold alone. It may be half of this without the intermediate layer. The Ti intermediate layer requires the smaller thickness of gold, whereas the Ag stick layer is the less active. In the red domain of wavelengths, the $\mathrm{Cr}$ layer influence differs from the $\mathrm{Ag}$ one. The optimization method gives the three unknown parameters $\left(e_{1}, e_{2}, \theta_{i}\right)$ simultaneously for each wavelength, and Eq. 3 enables to check the result of the optimization. The optimal intermediate thickness is plotted in Fig. 2(b). We can remark that $e_{1}$ may reach $16 \mathrm{~nm}$ and is not the smallest as possible. It is an increasing function of the wavelength and is always greater than 2 or $3 \mathrm{~nm}$, thicknesses that are commonly used in experiments. The whole system must be considered, and the intermediate layer contributes to the quality of the plasmon excitation. The thicknesses of Chromium or Titanium are almost the same along the spectrum, on the contrary of silver, for which it is smaller. Figure 2(c) gives the best incidence angles. In some experimental setups, the divergence of the p-polarized incoming wave may be of the same order of magnitude as the variations of $\theta_{i}$ along the visible spectrum. This parameter seems to be noncritical.

In order to discuss the influence of the various metals $M$ in the intermediate layer, we show the relative difference between dispersion curves $C\left(u, \lambda_{0}\right)$ for the best values of thicknesses $\left(e_{1}, e_{2}\right)$ in Fig. 3:

$C\left(u, \lambda_{0}\right)=\frac{R_{\mathrm{Au}-M}-R_{\mathrm{Au}}}{R_{\mathrm{Au}-M}+R_{\mathrm{Au}}}$ 


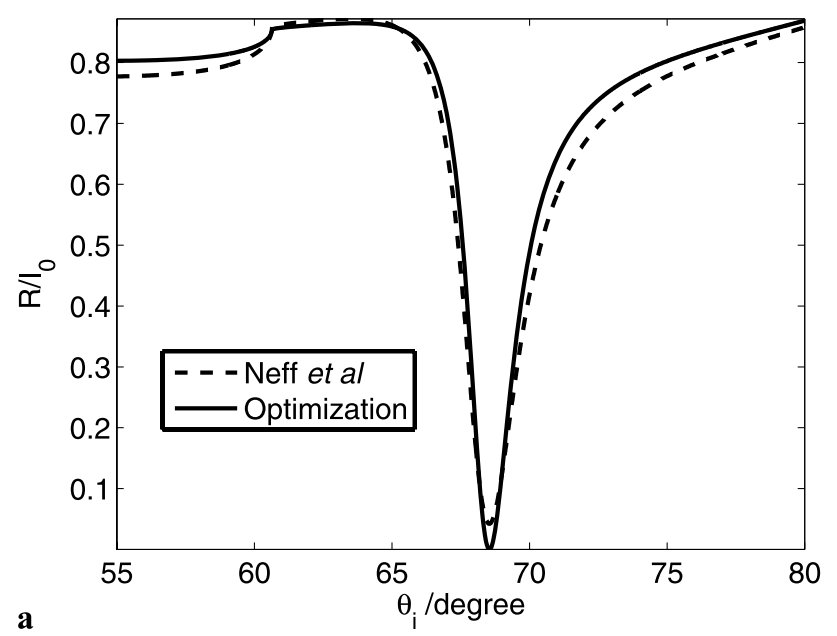

a

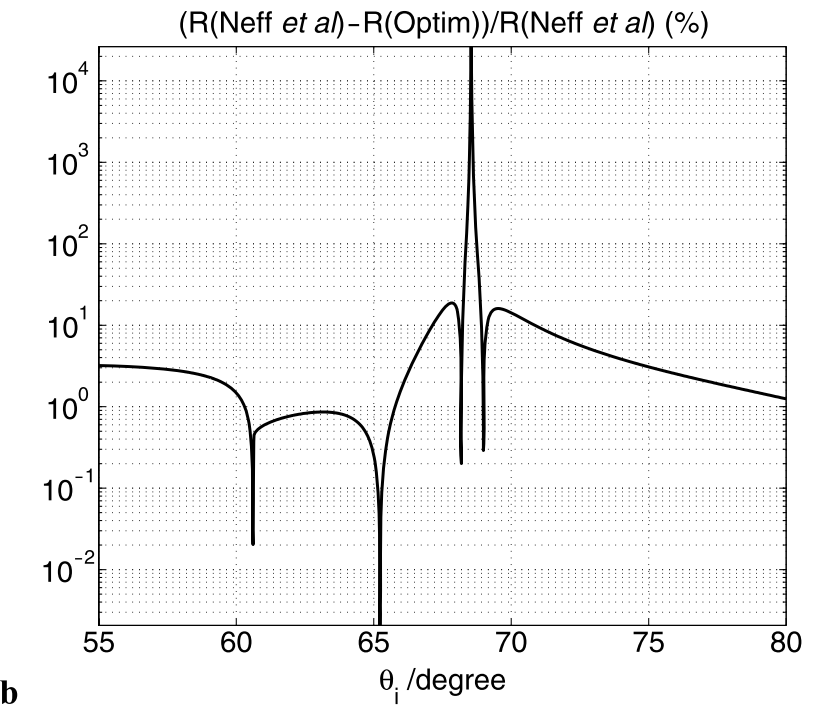

b

Fig. 4 (a) The same computation of SPR $\left(R / I_{0}\right)$ as in Ref. [8] (dashed line), and for optimized parameters (solid line). (b) The relative difference between the two curves in (a) expressed in percent and plotted with logscale along vertical direction

The visual inspection of these gray level maps exhibits both the decay and the widening of the SPR when the intermediate layer is considered in the computation for the best parameters $\left(e_{1}, e_{2}\right)$ (Fig. 2). The difference of two dispersion curves shows the red-shift of the plasmon as well as the best quality of the Ag intermediate layer to produce SPR close to that obtained for the Au alone.

To finish, in Fig. 4(a-b), the SPR $\left(R / I_{0}\right)$ is plotted in the same conditions as in Ref. [8] at $\lambda_{0}=670 \mathrm{~nm}$ with $e_{1}=$ $2 \mathrm{~nm}$ and $e_{2}=50 \mathrm{~nm}$. The permittivities are $\epsilon_{1}=2.329$, $\epsilon_{2}=13.45+8.74 j, \epsilon_{3}=-14.33+0.765 j$, and $\epsilon_{4}=1.769$, respectively. After optimization, the best values of the thicknesses are $e_{1}=2 \mathrm{~nm}$ and $e_{2}=56 \mathrm{~nm}$ inducing a sharper and narrower SPR value.

\section{Conclusion}

In this study, it has been shown that thin intermediate layer significantly modifies the conditions for the generation of a plasmon. The thickness of both the intermediate and gold layers must be controlled and depends strongly on the working wavelength. On the contrary, the plasmon optimization is hardly dependent on the angle of incidence. Such results would be of great interest in the optimization of nanogratings and nanosensors for both biological and physical applications. Moreover, a more complete study of sensitivity of sensors will be possible from the results of the same method of optimization for more complex geometries of sensors.

Acknowledgements Authors thank the Centre de Calcul Intensif ROMEO2 for computational facilities, the Région ChampagneArdennes and the Conseil Régional de l'Aube for financial supports.

\section{References}

1. E.M. Yeatman, Biosens. Biolectron. 11(6-7), 635 (1996)

2. P. Lecaruyer, M. Canva, J. Rolland, Appl. Opt. 46(12), 2361 (2007)

3. N. Bonod, S. Enoch, L. Li, E. Popov, M. Nevière, Opt. Express 11(5), 482 (2006)

4. C. Langhammer, Z. Yuan, I. Zoric, B. Kasemo, Nanoletters 6(4), 833 (2006)

5. A.I. Maaroof, G.B. Smith, Thin Solid Films 485, 198 (2005)

6. S. Xilian, S. Jianda, Appl. Surf. Sci. 253, 2093 (2006)

7. S. Ekgasit, C. Thammacharoen, F. Yu, W. Knoll, Appl. Spectrosc. 59(5), 661 (2005)

8. H. Neff, W. Zong, A.M.N. Lima, M. Borre, G. Holzhüter, Thin Solid Films 496, 688 (2006)

9. B.A. Sexton, B.N. Feltis, T.J. Davis, Sens. Actuator A-Phys. 141, 471 (2008)

10. E.D. Palik, Handbook of Optical Constants of Solid I (Academic Press, New York, 1985)

11. D. Barchiesi, E. Kremer, V.P. Mai, T. Grosges, J. Microsc. 229(3), 525 (2008)

12. D. Maystre, General Study of Grating Anomalies from Electromagnetic Surface Modes. Electromagnetic Surface Modes (Wiley, New York, 1982)

13. F. Abeles, Optics of Thin Films, in Advanced Optical Techniques, ed. by A.C.S. van Heel (North-Holland, Amsterdam, 1967), Chap. 5

14. M. Born, E. Wolf, Principles of Optics, 6th edn. (Pergamon, Oxford, 1992)

15. D. Barchiesi, Opt. Commun. 126, 7 (1996)

16. L. Li, J. Opt. Soc. Am. A 11(11), 2816 (1994)

17. J.M. Vigoureux, J. Opt. Soc. Am. A 9(8), 1313 (1992)

18. G. Granet, B. Guizal, J. Opt. Soc. Am. A 13, 1019 (1996)

19. J.B. Pendry, J. Mod. Opt. 41(2), 209 (1994)

20. E. Popov, L. Mashev, D. Maystre, Opt. Acta 33(5), 607 (1986)

21. H.P. Schwefel, Evolution and Optimum Seeking (Wiley, New York, 1995)

22. D. Macías, D. Barchiesi, Opt. Lett. 30, 2557 (2005) 